\title{
The $J_{1}-J_{2}$ antiferromagnet on the square lattice with Dzyaloshinskii-Moriya interaction : An exact diagonalization study
}

\author{
Andreas Voigt and Johannes Richter \\ Institut für Theoretische Physik, Otto-von-Guericke-Universität Magdeburg \\ Postfach 4120, D-39016 Magdeburg, Germany
}

(Received October 16, 2018)

\begin{abstract}
We examine the influence of an anisotropic interaction term of Dzyaloshinskii-Moriya (DM) type on the groundstate ordering of the $J_{1}-J_{2}$ spin- $\frac{1}{2}$-Heisenberg antiferromagnet on the square lattice. For the DM term we consider several symmetries corresponding to different crystal structures. For the pure $J_{1}-J_{2}$ model there are strong indications for a quantum spin liquid in the region of $0.4 \lesssim J_{2} / J_{1} \lesssim 0.65$. We find that a DM interaction influences the breakdown of the conventional antiferromagnetic order by i) shifting the spin liquid region, ii) changing the isotropic character of the groundstate towards anisotropic correlations and iii) creating for certain symmetries a net ferromagnetic moment.
\end{abstract}

\section{INTRODUCTION}

The two-dimensional quantum spin Heisenberg antiferromagnet (AFM) has attracted a considerable interest in connection with the magnetic properties of the high-temperature superconductors in recent time [1]. The $\mathrm{CuO}$ planes being responsible for the superconductivity show a strong in-plane exchange and only a small off-plane exchange [2]. Therefore in the insulating phase the interacting $\mathrm{Cu}$ spins should be well described by a two-dimensional spin- $\frac{1}{2}-$ Heisenberg antiferromagnet. There are several arguments [3 6] that additional to the nearest neighbour exchange $J_{1}$ a frustrating diagonal $J_{2}$ bond is relevant. The groundstate properties of this so-called $J_{1}-J_{2}$ model are widely discussed in the last years, mainly with respect to a possible breakdown of the magnetic long-range order (LRO) due to the combined influence of quantum fluctuations and frustration (see e.g. [7]- [17]). One finds evidence for a finite parameter region around $J_{2} / J_{1} \sim 0.5$ where quantum fluctuations open a window of a spin liquid phase. Moreover, there are indications of enhanced exotic order parameters (spin-Peierls, chiral) [8, 10, 14, 18, 19].

However, there are also indications for additional anisotropic terms in the Hamiltonian which could explain the experimentally observed weak ferromagnetism for instance in $\mathrm{La}_{2} \mathrm{CuO}_{4}$ [20,21. In general, a small ferromagnetic moment in antiferromagnets may appear in materials with low crystal symmetry. This tilting of the spins can be described by adding the so-called anisotropic Dzyaloshinskii-Moriya (DM) interaction to the isotropic Heisenberg model. Already 1957 Dzyaloshinskii formulated a phenomenological theory of these facts [22]. Three years later Moriya developed the microscopic theory of the weak ferromagnetism [23]. The occuring additional interaction term in the Hamiltonian is proportional to the DM vector $\vec{D}$. In some recent publications 224 28] one has examined the origin and the structure of $\vec{D}$. In this paper we discuss the influence of DM terms on the groundstate properties of the $J_{1}-J_{2}$ model on the square lattice.

\section{ANISOTROPIC SPIN INTERACTION}

Let us start with a general Hamiltonian, describing quadratic spin-spin interaction:

$$
\hat{H}=\sum_{i, j=1}^{N} \vec{S}_{i}^{T} \quad \hat{J}_{i, j} \quad \vec{S}_{j}
$$

with $\hat{J}_{i, j}$ being a $3 \times 3$ matrix of interaction, which can be written as

$$
\hat{J}=\frac{1}{3}(S p \hat{J}) \hat{1}+\hat{A}+\hat{M}
$$

where $\hat{1}$ is the unit matrix, $\hat{A}$ is antisymmetric and $\hat{M}$ is symmetric and traceless. With (2) equation (11) reads: 


$$
\hat{H}=\sum_{i, j=1}^{N}\left\{\frac{1}{3}\left(S p \hat{J}_{i, j}\right) \vec{S}_{i} \vec{S}_{j}+\vec{D}_{i, j}\left(\vec{S}_{i} \times \vec{S}_{j}\right)+\vec{S}_{i}^{T} \hat{M}_{i, j} \vec{S}_{j}\right\},
$$

where the first term is the isotropic Heisenberg interaction, the second one - the Dzyaloshinskii-Moriya interaction and the last one - the anisotropic pseudo-dipole interaction. The vector $\vec{D}_{i, j}$ contains the three independent components of $\hat{A}_{i, j}$. In general the occurrence of $\vec{D}_{i, j}$ requires low crystal symmetries. The origin of the anisotropic interaction $\vec{D}_{i, j}$ and $\hat{M}_{i, j}$ is the spin-orbit coupling. In the last few years there is some effort concerning the question of including the term with $\hat{M}_{i, j}$ in the calculations [29], but in what follows we neglect $\hat{M}_{i, j}$, because it is only of second order in the spin-orbit coupling constant $\lambda$ whereas $\vec{D}_{i, j}$ is of first order in $\lambda$.

The weak ferromagnetic moment in the predominantly antiferromagnetic ordered $\mathrm{CuO}$ planes in $\mathrm{La}_{2} \mathrm{CuO}_{4}$ can be interpreted with a small spin canting. This spin canting could be described via a DM interaction term. In 24] a general form of the DM vector is introduced and it is shown, that only a vector $\vec{D}_{i, j}$ which varies from bond to bond corresponds to the crystal structure and is able to describe the observed weak ferromagnetism. In 24,25] Coffey and coworkers consider different crystal symmetries and get $\vec{D}_{i, j}$ for the whole lattice by requiring for the vector $\vec{D}_{i, j}$ that the energy of any configuration of spins is invariant under the symmetry transformations of the crystal structure. Once $\vec{D}_{i, j}$ is fixed on a single bond, the symmetries determine $\vec{D}_{i, j}$ on the entire lattice.

Following [24,25] we consider in this paper two different symmetries for $\vec{D}_{i, j}$ which correspond to the orthorhombic and tetragonal phases of $\mathrm{La}_{2} \mathrm{CuO}_{4}$ 30 32]. The arrangements of the atoms in a CuO-plane are presented in figure 1. For the orthorhombic arrangement (figure 1a) one finds the following $\vec{D}_{i, j}$ :

- $\vec{D}_{A, B}=\left(d_{1}, d_{2}, 0\right)$

- $\vec{D}_{A, C}=\left(-d_{2},-d_{1}, 0\right)$

- $\vec{D}_{B, D}=-\vec{D}_{A, C}=\left(d_{2}, d_{1}, 0\right)$

- $\vec{D}_{C, D}=-\vec{D}_{A, B}=\left(-d_{1},-d_{2}, 0\right)$

- $\vec{D}_{C, E}=-\vec{D}_{A, C}=\left(d_{2}, d_{1}, 0\right)$

For the tetragonal arrangement (figure 1b) the $\vec{D}_{i, j}$ is as follows:

- $\vec{D}_{A, B}=\left(0, d_{1}, 0\right)$

- $\vec{D}_{A, C}=\left(0, d_{2}, 0\right)$

- $\vec{D}_{B, D}=-\vec{D}_{A, C}=\left(0,-d_{2}, 0\right)$

- $\vec{D}_{C, D}=-\vec{D}_{A, B}=\left(0,-d_{1}, 0\right)$

- $\vec{D}_{C, E}=-\vec{D}_{A, C}=\left(0,-d_{2}, 0\right)$

Furthermore we will distinguish between equal and different signs of the parameters $d_{1}$ and $d_{2}$. Following the arguments of [24] we will restrict our considerations to $d_{1}$ and $d_{2}$ of equal strength. Let us define four different cases for the parameters: orthorhombic symmetry with either $d_{1}=+d_{2}$ or $d_{1}=-d_{2}$ and tetragonal symmetry with either $d_{1}=+d_{2}$ or $d_{1}=-d_{2}$.

First let us consider the classical Hamiltonian

$$
\hat{H}_{D M}=\sum_{i, j=1}^{N} \vec{D}_{i, j}\left(\vec{S}_{i} \otimes \vec{S}_{j}\right)
$$

with the configurations for $\vec{D}_{i, j}$ as given above. $\vec{S}_{i}$ and $\vec{S}_{j}$ are classical $3 \mathrm{D}$ vectors and the summation runs over nearest neighbour bonds. The classical groundstate of (4) was discussed in detail in [24]. Here we briefly summarize some ground state features which are relevant for the further discussion. The energy of any nearest neighbour bond $(i, j)$ on the lattice can be minimized by spin vectors $\vec{S}_{i}$ and $\vec{S}_{j}$ perpendicular to each other and perpendicular to the DM vector $\vec{D}_{i j}$; the corresponding optimum bond energy is $E_{i j}=-\left|\vec{D}_{i j}\right| S^{2}$. Because of the special symmetry $\left|d_{1}\right|=\left|d_{2}\right|$ there is no frustration in the Hamiltonian (4) and the total groundstate configuration can be build by the suitable arrangement of optimized bonds. In table 1 we present some important groundstate features for the considered four different cases of $\vec{D}_{i, j}$. 


\begin{tabular}{c|llcc} 
case & $\begin{array}{llll}\text { crystal } \\
\text { structure }\end{array}$ & $\begin{array}{l}\text { DM } \\
\text { vector }\end{array}$ & $\begin{array}{c}\text { net } \\
\text { FM }\end{array}$ & $\begin{array}{c}\text { spin } \\
\text { configuration }\end{array}$ \\
\hline Ia & orthorhombic & $d_{1}=+d_{2}=d$ & no & $4 \mathrm{SL}$ \\
Ib & orthorhombic & $d_{1}=-d_{2}=d$ & yes & $2 \mathrm{SL}$ \\
IIa & tetragonal & $d_{1}=+d_{2}=d$ yes & $2 \mathrm{SL}$ \\
IIb & tetragonal & $d_{1}=-d_{2}=d$ & no & $4 \mathrm{SL}$
\end{tabular}

Table 1: The four considered cases with some of its groundstate features of the classical DM Hamiltonian $\hat{H}_{D M}$

Here FM means ferromagnetic moment and 2 SL or 4 SL means 2 or 4 sublattices, respectively. As an example the groundstates for the cases Ia and Ib are illustrated in more detail in figure 2.

In the next section we turn to the quantum system.

\section{THE MODEL}

We start with the so called $J_{1}-J_{2}$ model on the square lattice:

$$
\hat{H}_{J_{1}, J_{2}}=\sum_{i=1}^{N} J_{1}\left(\vec{S}_{i} \vec{S}_{i+\vec{x}}+\vec{S}_{i} \vec{S}_{i+\vec{y}}\right)+J_{2}\left(\vec{S}_{i} \vec{S}_{i+\vec{x}+\vec{y}}+\vec{S}_{i} \vec{S}_{i-\vec{x}+\vec{y}}\right) .
$$

$\vec{S}_{i}$ denotes the spin- $\frac{1}{2}$ operator on site i and $\vec{x}$ and $\vec{y}$ are the unit lattice vectors in x- and y-direction.

An intensive investigation over the last years (see e.g. [7]- 17]) suggests the following phase diagram: For small values of $J_{2} / J_{1}$ the system shows AFM-LRO. At some critical value $J_{2}^{\text {crit }} / J_{1}$ of about $\simeq 0.4$ the conventional collinear AFM-LRO breaks down. Then a region of a spin-liquid state could be realized for $0.4 \leq J_{2} / J_{1} \leq 0.65$. For values of $J_{2} / J_{1} \geq 0.65$ an AFM-LRO arises within the initial sublattices (4-sublattice AFM).

Now we add the Hamiltonian of the DM-interaction

$$
\begin{gathered}
\hat{H}=\hat{H}_{J_{1}, J_{2}}+\hat{H}_{D M} \\
\hat{H}_{D M}=\sum_{i=1}^{N}\left[\vec{D}_{i, i+\vec{x}}\left(\vec{S}_{i} \times \vec{S}_{i+\vec{x}}\right)+\vec{D}_{i, i+\vec{y}}\left(\vec{S}_{i} \times \vec{S}_{i+\vec{y}}\right)\right] .
\end{gathered}
$$

Using spin-flip operators we can rewrite the DM term

$$
\begin{aligned}
\vec{D}_{i, j}\left(\vec{S}_{i} \times \vec{S}_{j}\right) & =\frac{1}{2}\left\{i D_{i j}^{x}\left[S_{i}^{-} S_{j}^{z}-S_{i}^{+} S_{j}^{z}-S_{i}^{z} S_{j}^{-}+S_{i}^{z} S_{j}^{+}\right]\right. \\
& -D_{i j}^{y}\left[S_{i}^{-} S_{j}^{z}-S_{i}^{z} S_{j}^{+}-S_{i}^{z} S_{j}^{-}+S_{i}^{+} S_{j}^{z}\right] \\
& \left.+i D_{i j}^{z}\left[S_{i}^{+} S_{j}^{-}-S_{i}^{-} S_{j}^{+}\right]\right\}
\end{aligned}
$$

The Hamiltonian $H_{D M}$ contains terms of the form $S_{i}^{ \pm} S_{j}^{z}$ which do not commute with the z-component of the total spin $\vec{S}$. Hence we have to take all $2^{N}$-Ising states for the construction of the wave function. We use a modified Lanczos procedure to calculate the groundstate of the system.

\section{NUMERICAL RESULTS}

Based on the properties of the pure $J_{1}-J_{2}$ model we discuss several order parameters, cf. [8, 11, 14, which describe the relevant magnetic properties of the model.

For dominating $J_{1}$ the corresponding antiferromagnetic LRO parameters are the components of the square of the staggered magnetization:

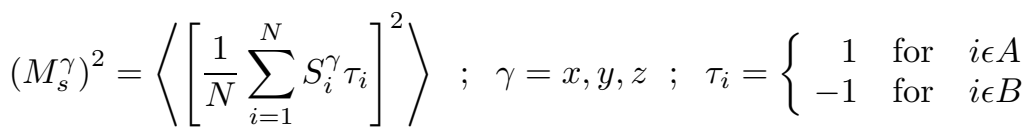


where $S_{i}^{\gamma}$ is the $\gamma$-component of the spin on site $\mathrm{i}, \tau_{i}$ is the corresponding staggered factor and $\mathrm{A}$ and B are the two sublattices. Obvious these parameters describe the ordinary 2-sublattice antiferromagnetic ordering.

For dominating $J_{2}$ the relevant order parameters are

$$
\left(M_{s, \alpha}^{\gamma}\right)^{2}=\left\langle\left[\frac{1}{N / 2} \sum_{i_{\alpha}=1}^{N / 2} S_{i}^{\gamma} \tau_{i, \alpha}\right]^{2}\right\rangle ; \gamma=x, y, z ; \tau_{i, \alpha}= \pm 1 ; \alpha=A, B .
$$

The sum runs over the sites of the sublattice $\alpha$. These order parameters describe antiferromagnetic ordering within the two initial sublattices A and B, when the whole system forms a 4-sublattice AFM. For the rotational invariant model without DM interaction the x,y,z components of the respective parameters are identical, but the DM interaction breaks this rotational symmetry.

For $\vec{D} \neq 0$ a weak ferromagnetism may occur. The relevant parameters for describing this weak ferromagnetism are the components of the square of the magnetization,

$$
\left(M^{\gamma}\right)^{2}=\left\langle\left[\frac{1}{N} \sum_{i=1}^{N} S_{i}^{\gamma}\right]^{2}\right\rangle ; \gamma=x, y, z
$$

Obviously $\sum_{\gamma}\left(M^{\gamma}\right)^{2}$ is the expectation value of the square of the total spin $\left\langle\vec{S}^{2}\right\rangle$.

In the pure model $\left(\vec{D}_{i, j}=0\right)$ the Hamiltonian (6) commutes with $\vec{S}^{2}$ and $S^{z}$ and the groundstate is isotropic with $\vec{S}^{2}=0$ (and consequently $\left(M^{\gamma}\right)^{2}=0$ ). If we now put on the anisotropic DM interaction $\left(\vec{D}_{i, j} \neq 0\right)$ the Hamiltonian does not commute with $\vec{S}^{2}$ and we may expect $\left(M^{\gamma}\right)^{2} \neq 0$. Koshibae et al [27] pointed out that anisotropic spin correlations are a result of the interplay between quantum fluctuations and the anisotropic DM interaction. This can be illustrated as follows: Consider a classical spin system without DM interaction and a collinear spin structure. The DM term contains the cross product of the spins so it vanishes. If now the quantum fluctuations are put on, the DM interaction favours the direction of spin fluctuations with minimal energy. Of course this direction depends on the DM vector but additionally on the classical collinear structure.

We have calculated the groundstate for a square lattice with $N=16$ and $N=20$ sites for the four different configurations of the DM vector given in table 1. In the following we focus our interest on the orthorhombic symmetry, since this symmetry is valid for low doping in $\mathrm{La}_{2-x} \mathrm{Ba}_{x} \mathrm{CuO}_{4} 32$. Furthermore we present preferably the data for $N=20$. The corresponding data for $N=16$ are qualitatively the same. For simplicity we put $J_{1}=1$ for the rest of the paper.

First we consider the situations of $J_{2}=0$ and of $J_{2}=1$. Then the isotropic model $\left(\vec{D}_{i, j}=0\right)$ is collinear long-range ordered with either a 2 -sublattice structure $\left(J_{2}=0\right)$ or a 4 -sublattice structure $\left(J_{2}=1\right)$. The influence of the DM interaction on the magnetic order parameters is shown in figures 3 and 4 .

Figure 3 shows the different components of the ferromagnetic moment $\left(M^{\gamma}\right)^{2}$ as a function of the DM interaction for $J_{2}=0$. The results are as follows: The DM interaction causes a small ferromagnetic moment $\left(\left(M^{\gamma}\right)^{2} \neq 0\right)$ which increases with $d$. This moment is anisotropic: $\left(\left(M^{z}\right)^{2}>\left(M^{x, y}\right)^{2}\right.$. The ferromagnetic moment is maximal for case $\mathrm{Ib}$ which is in accordance with the classical situation (see table 1). We have calculated the same parameters for $J_{2}=1$. In that case the classical structure is a 4-sublattice AFM, which does not have a net ferromagnetic moment. Consequently we find in the quantum case that the ferromagnetic moment is about 10 times smaller as for $J_{2}=0$.

In the next two figures $4 \mathrm{a}$ and $4 \mathrm{~b}$ we present the different components of the AFM-LRO parameters. For $J_{2}=0$ the 2 sublattice AFM-LRO parameters $\left(M_{s}^{\gamma}\right)^{2}$ have to be considered and for $J_{2}=1$ the 4-sublattice AFM-LRO parameters $\left(M_{s, \alpha}^{\gamma}\right)^{2}$ are relevant. As discussed above the anisotropy depends on the DM vector $\vec{D}_{i, j}$ and on the underlying magnetic structure. Correspondingly the anisotropy is changed going from $J_{2}=0$ to $J_{2}=1$ or, alternatively, going from case Ia to case Ib. Furthermore, in dependence on the symmetry of the DM vector, we can observe either an enhancement or a suppression of a certain component of the antiferromagnetic order parameter. For instance the z-component of the 4-sublattice AFM-LRO parameter $\left(M_{s, \alpha}^{z}\right)^{2}$ is enhanced by the DM interaction if $\vec{D}_{i, j}$ favours a classical 4-sublattice structure (see figure $4 \mathrm{a}$ ) whereas the same order parameter is suppressed if $\vec{D}_{i, j}$ favours a classical 2-sublattice structure (see figure $4 \mathrm{~b}$ ).

The comparison of the magnitude of ferromagnetic and antiferromagnetic order parameters (figures 3 and 4) shows that the ferromagnetic moment remains small even if the DM interactions reaches the same strength as the exchange coupling.

Let us now discuss the antiferromagnetic order parameters in dependence on the frustration parameter $J_{2} / J_{1}$. For this we select from figure 4 in each case the dominating components $\left(M_{s}^{\gamma}\right)^{2}$ and $\left(M_{s, \alpha}^{\gamma}\right)^{2}$. The magnetic order for 
$\vec{D}_{i, j}=0$ is determined by the competition between $J_{1}$ and $J_{2}$. For dominating $J_{1}$ and for dominating $J_{2}$ the magnetic structure is collinear antiferromagnetic with two or four sublattices. The transition between both phases is in the classical case at $J_{2}=\frac{1}{2} J_{1}$ and may be connected with a spin liquid phase in the quantum case. Since the DM term also favours a two (case Ib) or a four sublattice structure (case Ia) we expect that this competition is influenced if we put on the DM term. The result should be a shift of the transition either to lower or higher values of $J_{2}$.

As shown in figure 5 we find indeed the expected shift, it is in particular strong for case Ia. As discussed above, the critical $J_{2}$ for the pure model $\left(\vec{D}_{i, j}=0\right)$ is about $J_{2} \approx 0.4$, which is too large to be realistic for the cuprate superconductors. We conclude that a DM interaction of this symmetry is able to support the breakdown of the antiferromagnetic long-range order due to frustration and to shift the critical $J_{2}$ to more realistic values. (Notice, that the abruptness of the change in the order parameters seen in figure 5 as well as in figure 6 is due to the well-known level crossing for the $N=20$ lattice (see e.g. [8], [10])).

Finally we will discuss the region of a possible spin liquid in more detail. As pointed out in [8, 10, 14, 18, there are two interesting candidates for unconventional noncollinear ordering in this region. One is a vector chiral order parameter introduced in 10,14,

$$
\begin{aligned}
C^{\gamma} & =\left\langle\frac{1}{3}\left[\frac{1}{2 N} \sum_{i=1}^{N}\left(\vec{C}_{i, i+\vec{x}, i+\vec{x}+\vec{y}}^{\gamma}-\vec{C}_{i, i+\vec{x}+\vec{y}, i+\vec{y}}^{\gamma}\right)\right]^{2}\right\rangle \\
\vec{C}_{i j l}^{\gamma} & =8 \vec{e}_{\gamma}\left(\left(\vec{S}_{i} \times \vec{S}_{j}\right)+\left(\vec{S}_{j} \times \vec{S}_{l}\right)+\left(\vec{S}_{l} \times \vec{S}_{i}\right)\right) .
\end{aligned}
$$

This order parameter measures the handiness of a plaquette of three spins. For the isotropic Heisenberg system this parameter is the same for all three components $\mathrm{x}, \mathrm{y}$ and $\mathrm{z}$. But in our case of anisotropic DM interaction the different components may have different values.

Another candidate for exotic ordering is the dimer (or spin-Peierls) order parameter,

$$
D=2\left[\frac{1}{N} \sum_{i=1}^{N}(-1)^{i_{\vec{x}}} \vec{S}_{i} \vec{S}_{i+\vec{x}}\right]^{2} .
$$

which measures the long-range phase coherence of spin dimers (singlets of two spins) along the $\mathrm{x}$ (or y) direction.

In figure 6 we present these parameters $C^{\gamma}$ and $D$, where we have selected the largest component of $C$. Both order parameters show in the pure model a characteristic maximum at about $J_{2}=0.55$. The influence of the DM interaction is twofold. First the maxima are shifted in the same way as discussed for figure 5. Second the maxima are suppressed particularly for $D$. The suppression of the maximum in $D$ can be simply understood by having in mind that the dimer ordering is connected with a singlet groundstate which is obviously not realized for $\vec{D}_{i, j} \neq 0$. On the other hand, the effect of the DM interaction on the chiral order parameter $C^{\gamma}$ seems to be more complicated. The DM term creates a spin canting which might under certain circumstances support the realization of a vector chiral ordering. Indeed, we found one example $\left(N=16, d_{1}=+d_{2}=0.3\right)$, where the chiral order parameter is enhanced in a small region around $J_{2} \approx 0.4$ (figure $6 \mathrm{c}$ ). Here the DM interaction term together with the frustrating $J_{2}$ causes some canted groundstate with enhanced vector chirality. However, it remains unclear whether this increase of $C^{\gamma}$ is an artifact of the particular cluster symmetry of $N=16$.

\section{SUMMARY}

The magnetic properties of the $J_{1}-J_{2}$ model with anisotropic Dzyaloshinskii-Moriya (DM) interaction are determined by the competition between terms favouring a collinear 2-sublattice AFM and terms favouring a collinear 4 -sublattice AFM. The former one is stabilized by the $J_{1}$ term and the latter by the $J_{2}$ term. The DM term may favour the 2-sublattice structure as well as the 4-sublattice structure in dependence on the symmetry of the DM vector $\vec{D}_{i, j}$ (see table 1 in section 2). As a result the DM interaction shifts the spin liquid region (which separates the two collinear antiferromagnetic phases for small and large $J_{2}$ ) to larger or smaller values of $J_{2}$. Because the critical $J_{2}^{\text {crit }} \approx 0.4$ for the breakdown of the 2-sublattice LRO in the pure model $\left(\vec{D}_{i, j}=0\right)$ is much larger than realistic $J_{2}$ values for cuprate superconductors [4] additional mechanisms are needed to shift the transition to smaller values of $J_{2}$. Recently it has been shown 14 that static holes simulating doping can shift $J_{2}^{\text {crit }}$ in the desired direction. In this paper we find that a DM term with suitable symmetry acts in the same direction: In case of orthorhombic symmetry a DM vector with identical $\mathrm{x}$ - and $\mathrm{y}$-components (case 1a in table 1) is appropriate to weaken the 2 -sublattice antiferromagnet and to realize lower $J_{2}^{\text {crit }}$. 
Besides of the shift of the transition region the DM term creates an anisotropy in the spin correlations. Which components are enhanced and which are suppressed is a result of the interplay between quantum fluctuations, the particular symmetry of the DM vector as well as the underlying magnetic structure of the pure model. Hence it occurs that for the 2-sublattice AFM another component is favoured than for the 4-sublattice AFM.

The third remarkable effect of the DM interaction is the appearance of a weak ferromagnetic moment. For the considered finite lattices this moment occurs for all symmetries of $\vec{D}_{i, j}$. In accordance with the classical picture the orthorhombic structure with different x and y components of $\vec{D}_{i, j}$ (case Ib in table 1) yields the strongest ferromagnetic moment and might indicate the existence of weak ferromagnetism in the thermodynamic limit just for this symmetry of $\vec{D}_{i, j}$.

Finally we analyzed exotic dimer and vector chiral order parameters showing a characteristic maximum in the spin liquid region. This maximum is in general suppressed by the DM term.

Besides of the most important orthorhombic symmetry considered preferably in the paper we have also calculated the magnetic quantities for the tetragonal symmetry (cf. figure $1 \mathrm{~b}$ and table 1 ). In principle the same scenario as for

the orthorhombic symmetry is valid, i.e. in dependence on the strength and the symmetry of the DM vector $\vec{D}_{i, j}$ we may have anisotropy in the spin correlations, a net ferromagnetic moment and a shift of the spin liquid phase.

\section{ACKNOWLEDGMENT}

This work has been supported by the Deutsche Forschungsgemeinschaft (Project No. Ri 615/1-2).

[1] Manousakis E 1991 Rev. Mod. Phys. 63 p 1

[2] Shirane G, Endoh Y, Birgeneau R J, Kastner M A, Hidaka Y, Oda M, Suzuki M and Murakami T 1987 Phys. Rev. Lett. 59 p 1613

[3] Inui M, Doinach S and Gabay M 1988 Phys. Rev. B 38 p 6631

[4] Annett F, Martin R, McMahan A and Satpathy S 1989 Phys. Rev. B 40 p 2620

[5] Ihle D and Kasner M 1990 Phys. Rev. B 42 p 4760

[6] Schmidt H J and Kuramoto Y 1990 Physica C 167 p 263

[7] Chandra P and Doucot B 1988 Phys. Rev. B 38 p 9335

[8] Dagotto E and Moreo A 1989 Phys. Rev. Lett. 63 p 2148

[9] Chubukov A 1991 Phys. Rev. B 44 p 6861

[10] Richter J, Gros C and Weber W 1991 Phys. Rev. B 44 p 906

[11] Schulz H J and Ziman T A L 1992 Europhys. Lett. 18 p 355

[12] Ivanov N B and Ivanov P Ch 1992 Phys. Rev. B 46 p 8206

[13] Rastelli E and Tassi A 1992 Phys. Rev. B 46(17) p 10793

[14] Richter J 1993 Phys. Rev. B 47(10) p 5794

[15] Dotsenko A V and Sushkov O P 1994 Phys. Rev. B 50 p 13821

[16] Barabanov A F and Beresovsky V M 1994 J. Phys. Soc. Jpn. 63 p 3974

[17] Richter J, Ivanov N B and Retzlaff K 1995 J. Magn. Magn. Mater. 140-144 p 1609

[18] Singh R R P and Narayanan R 1990 Phys. Rev. Lett. 65 p 2590

[19] Kawarabayashi T and Suzuki M 1991 J. Phys. Soc. Jap. 60 p 825

[20] Thio T, Thurston T R, Preyer N W, Picone P J, Kastner M A, Jenssen H P, Gabbe D R, Chen C Y, Birgeneau R J and Aharony A 1988 Phys. Rev. B 38(1) p 905

[21] Cheong S-W, Thompson J D and Fisk Z 1989 Phys. Rev. B 39(7) p 4395

[22] Dzyaloshinskii I E 1958 J. Phys. Chem. Solids 4 p 241

[23] Moriya T 1960 Phys.Rev. 120(1) p 91

[24] Coffey D, Bedell K and Trugman S 1990 Phys. Rev. B 42(10) p 6509

[25] Coffey D, Rice T M and Zang F C 1991 Phys. Rev. B 44(18), p 10112

[26] Bonesteel N E, Rice T M and Zang F C 1991 Phys. Rev. Lett. 68(17) p 2648

[27] Koshibae W, Otha Y and Maekawa S 1991 Physica C 185-189 p 1509

[28] Feldkemper S 1991 diploma work Universität Dortmund

[29] Shektman L, Entin-Wohlmann O and Aharony A 1992 Phys. Rev. Lett. 69(5) p 836

[30] Longo J M and Raccah P M 1973 J. solid state chemistry 6 p 526 
[31] Grande B, Müller-Buschbaum H and Schweizer M 1977 Zeitschrift für anorganische allgemeine Chemie 428 p 120

[32] Axe J D, Moudden A H, Hohlwein D, Cox D E, Mohanty K M, Moodenbaugh A R and Youwen Xu 1989 Phys. Rev. Lett. 62(23) p 2751

FIG. 1. The orthorhombic (a) and tetragonal (b) arrangements of the CuO-octahedra taken from [24]. The filled circles are the copper sites which carry the spins, the open circles are oxygen sites, which are tilted up out of the plane and the crossed circles are oxygen sites, which are tilted down out of the plane. The square represents one tilted CuO-octahedra. The points A-E denote certain copper sites used in the text. The arrows show one particular arrangement of the DM vector $\vec{D}_{i, j}$ for this lattice (cases Ia and IIa, see table 1.

FIG. 2. The classical spin configurations for the Hamiltonian (4); (a): case Ia with a 4-sublattice configuration and without a net ferromagnetic moment, (b): case Ib with a 2-sublattice configuration and with a net ferromagnetic moment. (All spins are aligned either along the z-axis or in the xy-plane.)

FIG. 3. The $\mathrm{x}, \mathrm{y}$ and $\mathrm{z}$ components of square of the magnetization $\left(M^{\gamma}\right)^{2}$ versus $d / J_{1}$ for case Ia (full line) and case Ib (dashed line) for $N=20$ and $J_{2}=0$.

FIG. 4. The 2-sublattice AFM-LRO parameters $\left(M_{s}^{\gamma}\right)^{2}$ for $J_{2}=0$ (full lines) and the 4-sublattice AFM-LRO parameters $\left(M_{s, \alpha}^{\gamma}\right)^{2}$ for $J_{2}=1$ (dashed lines) versus $d / J_{1}$ for $N=20$; (a): case Ia, (b): case Ib .

FIG. 5. The dominant 2-sublattice AFM-LRO parameters $\left(M_{s}^{\gamma}\right)^{2}$ (full lines) and the dominant 4-sublattice AFM-LRO parameters $\left(M_{s, \alpha}^{\gamma}\right)^{2}$ (dashed lines) versus $J_{2} / J_{1}$ for $N=20$ and different values of $d$; (a): case Ia with $\left(M_{s}^{x, y}\right)^{2}$ and $\left(M_{s, \alpha}^{z}\right)^{2}$, (b): case Ib with $\left(M_{s}^{z}\right)^{2}$ and $\left(M_{s, \alpha}^{x, y}\right)^{2}$.

FIG. 6. Exotic order parameters versus $J_{2} / J_{1}$ for different $d$ : (a) - The dominant chiral order parameter $C^{x, y}$ for case Ia (solid line) and $C^{z}$ for case Ib (dashed line) for $N=20$; (b) - The dimer order parameter $D$ for case Ia (solid line) and case Ib (dashed line) for $N=20$; (c) - The dominant chiral order parameter $C^{x, y}$ for case Ia (solid line) and $C^{z}$ for case Ib (dashed line) for $N=16$. 




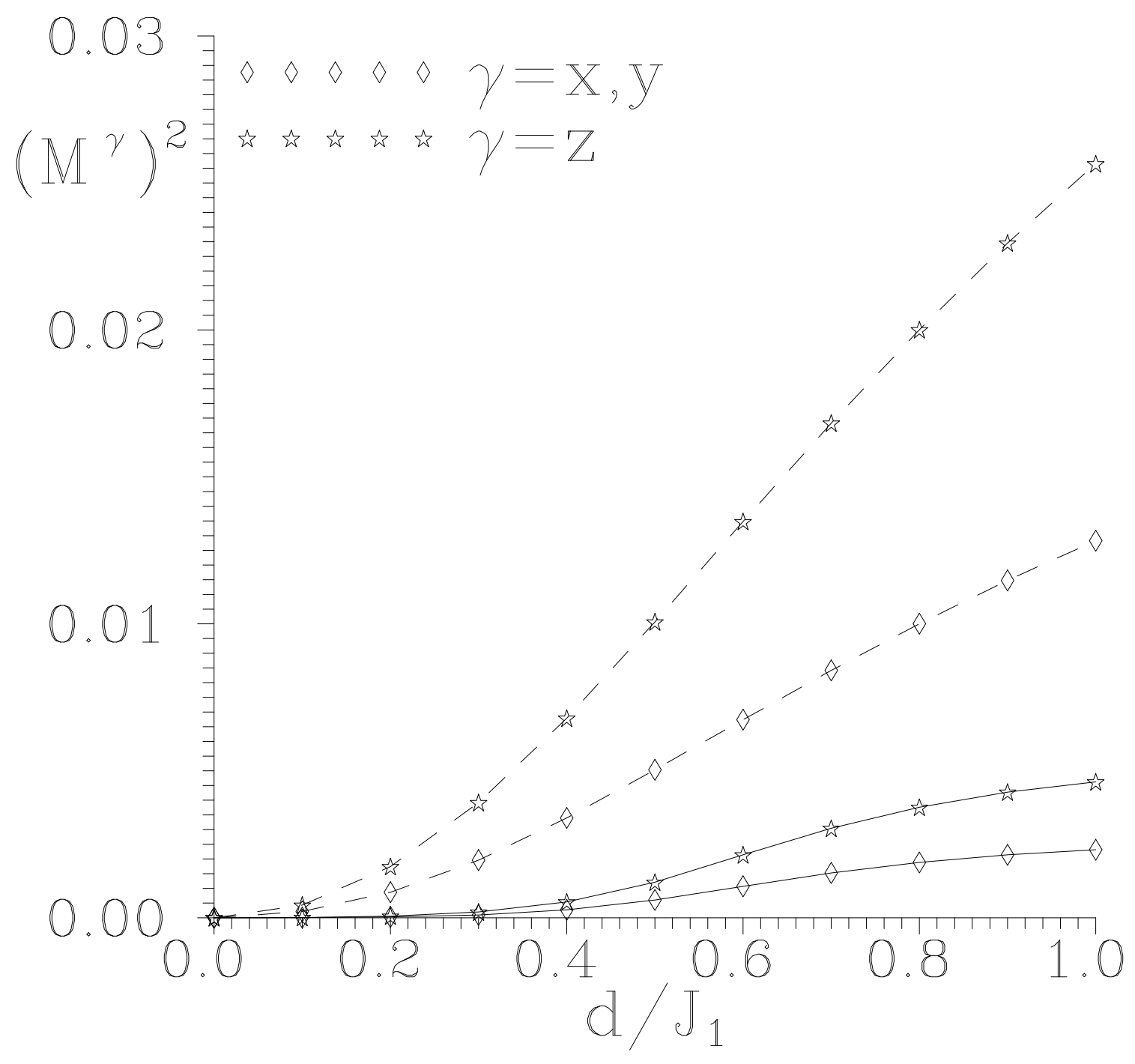




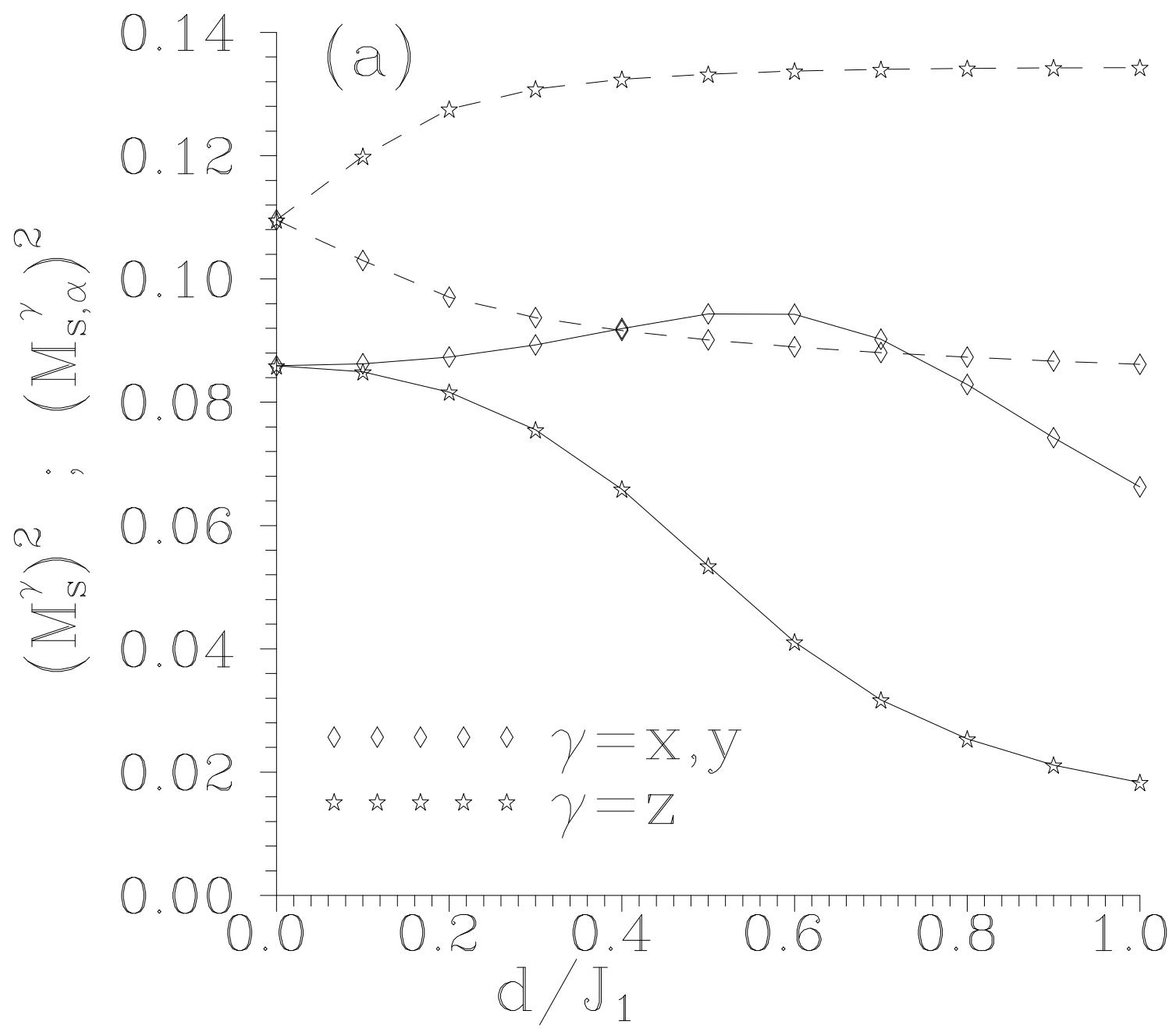




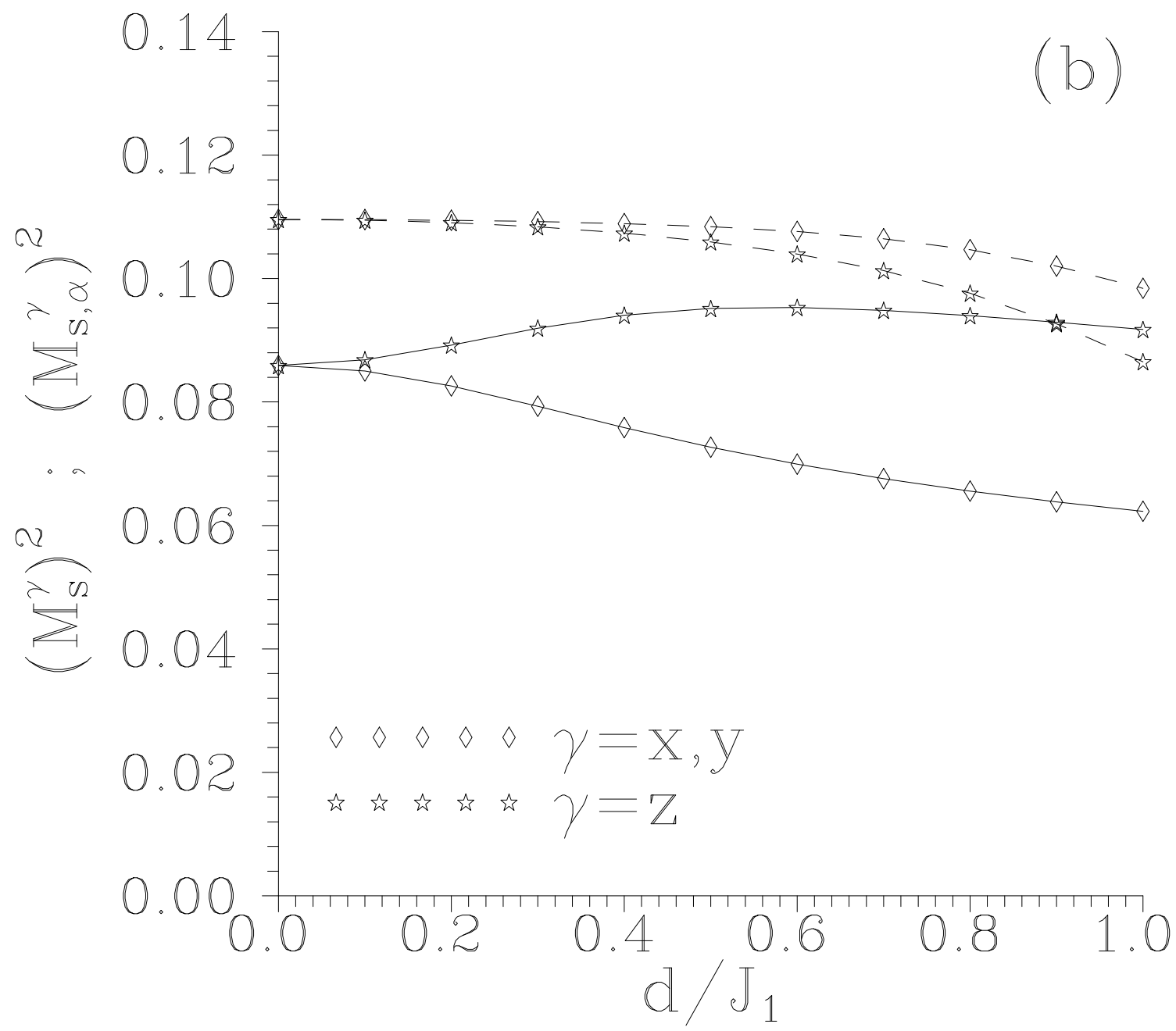




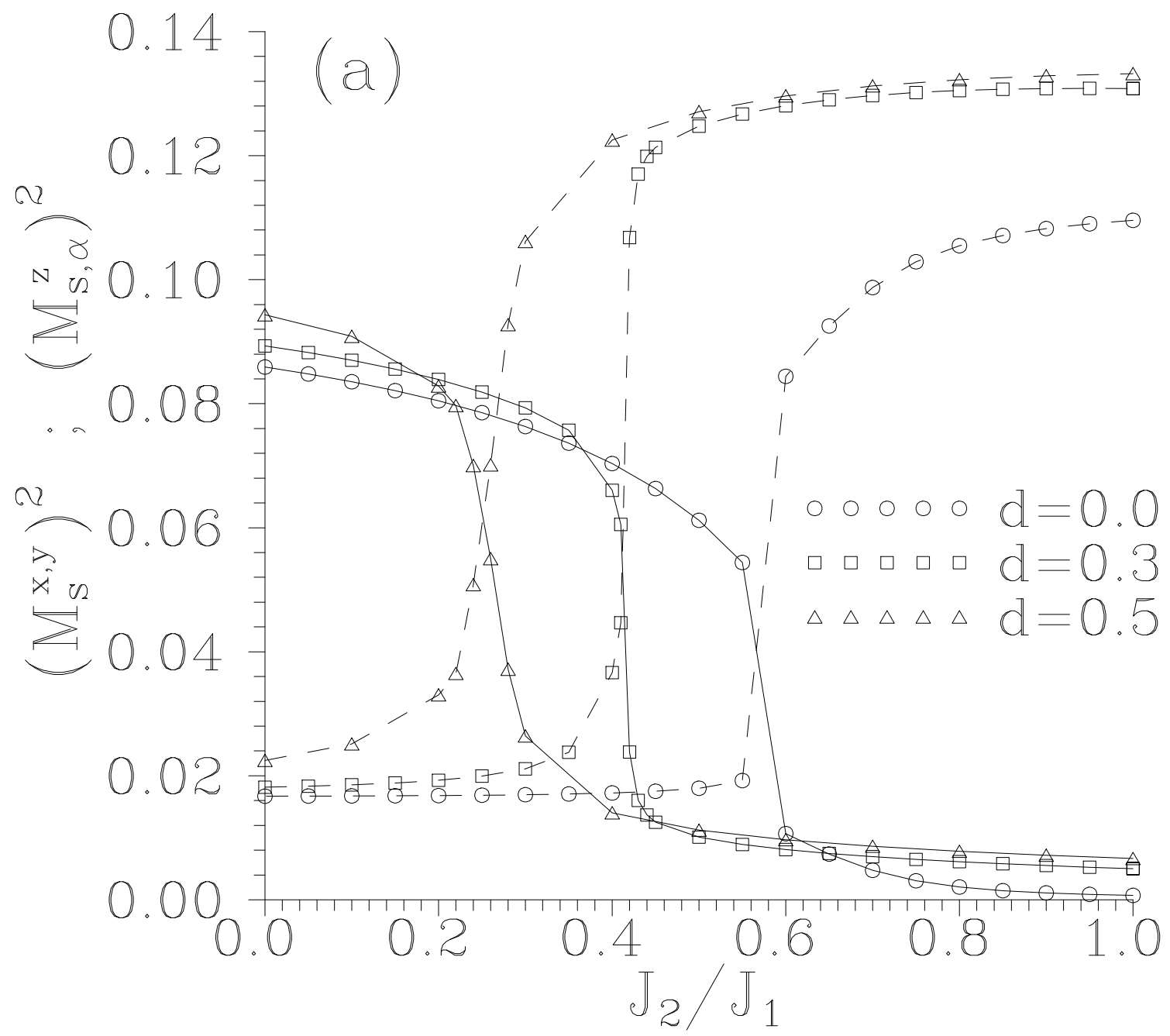




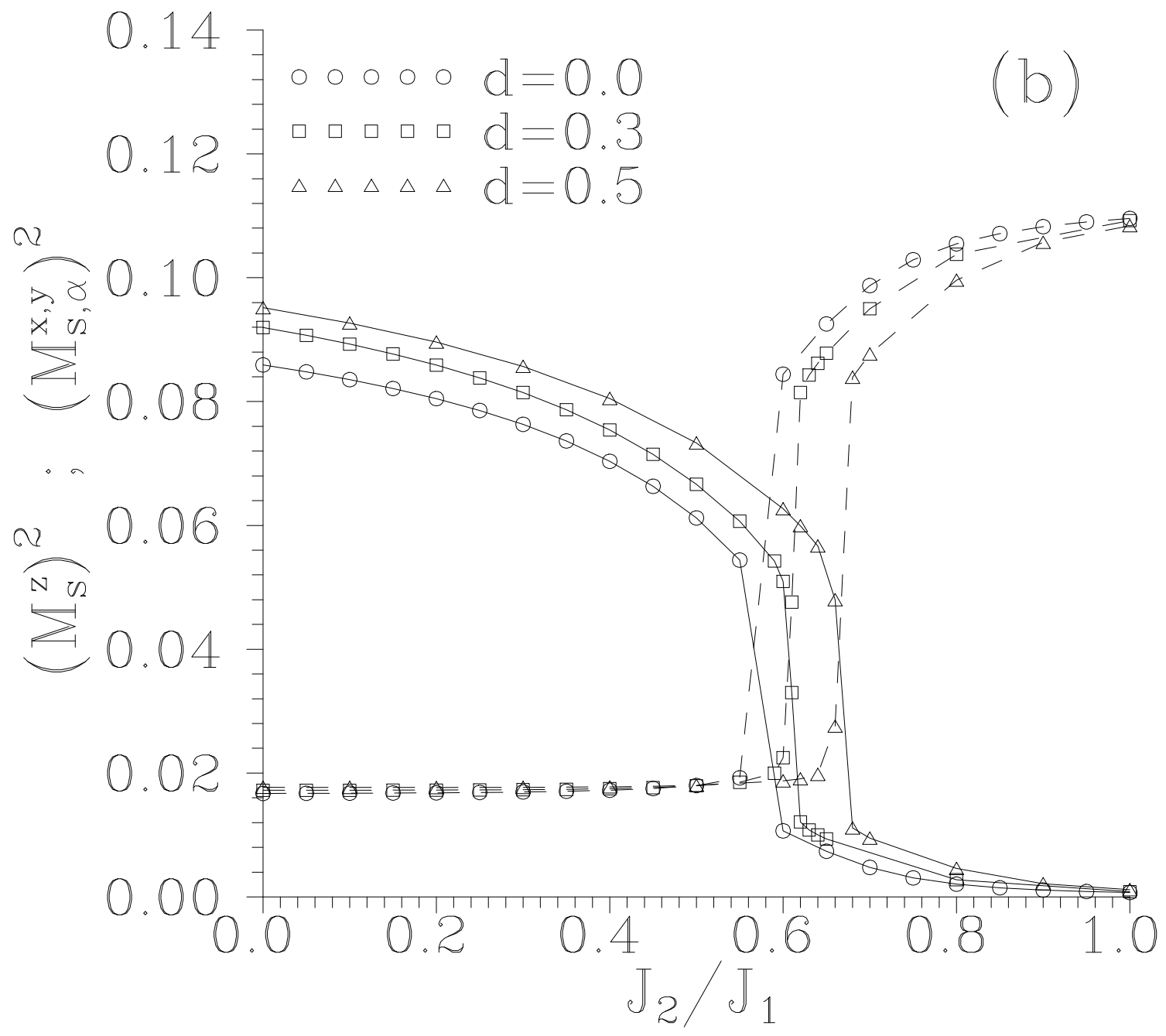




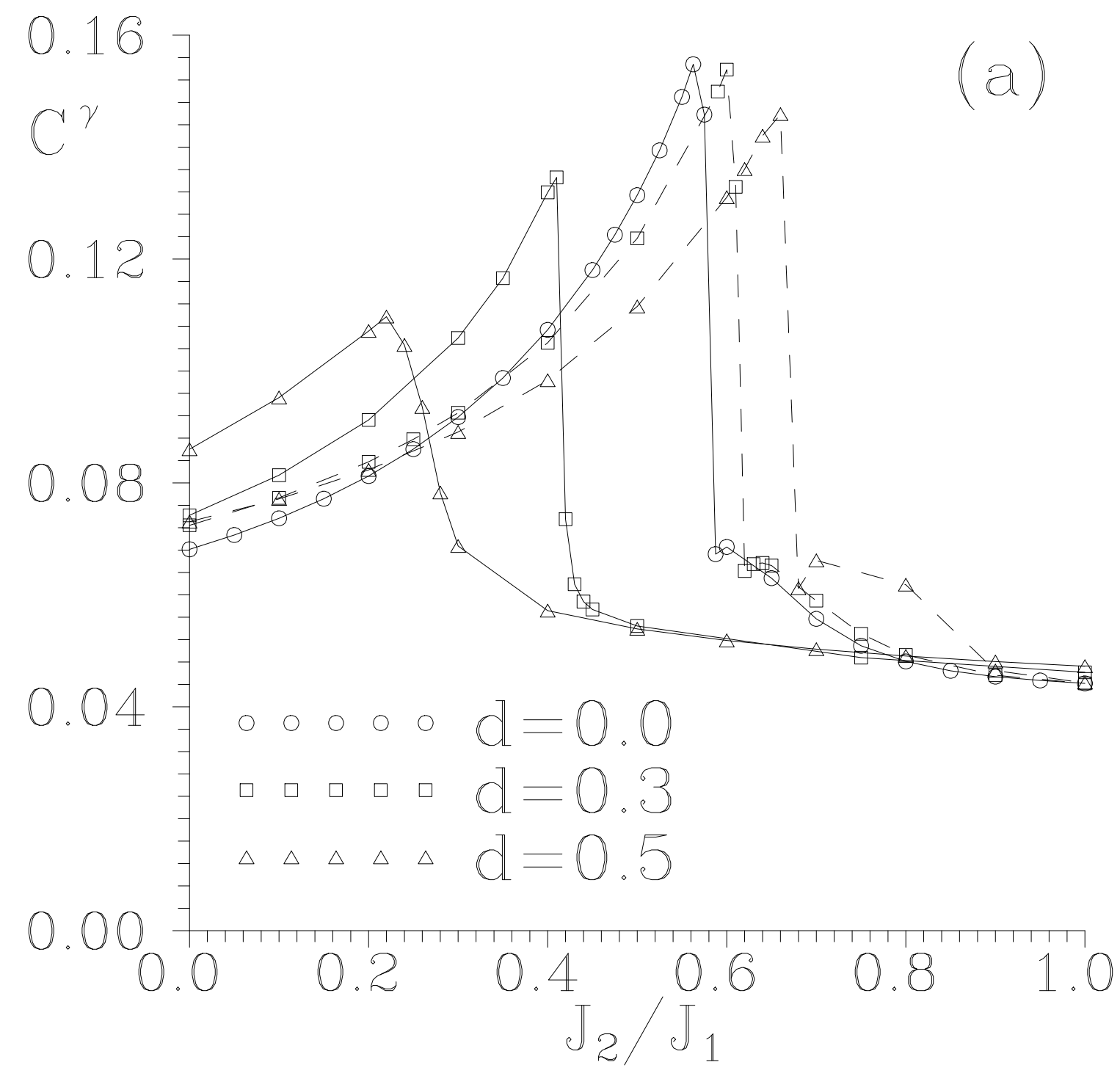




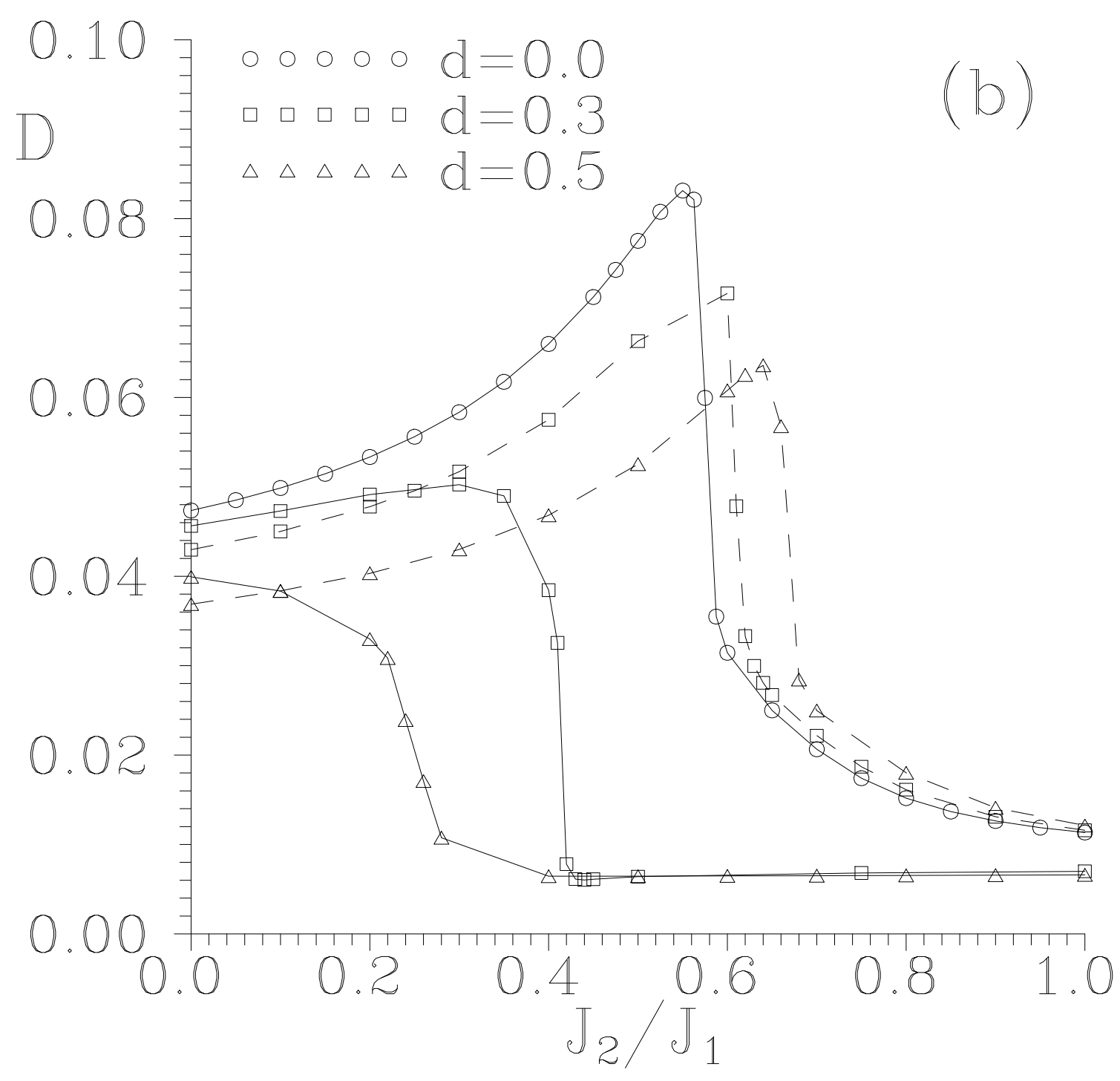




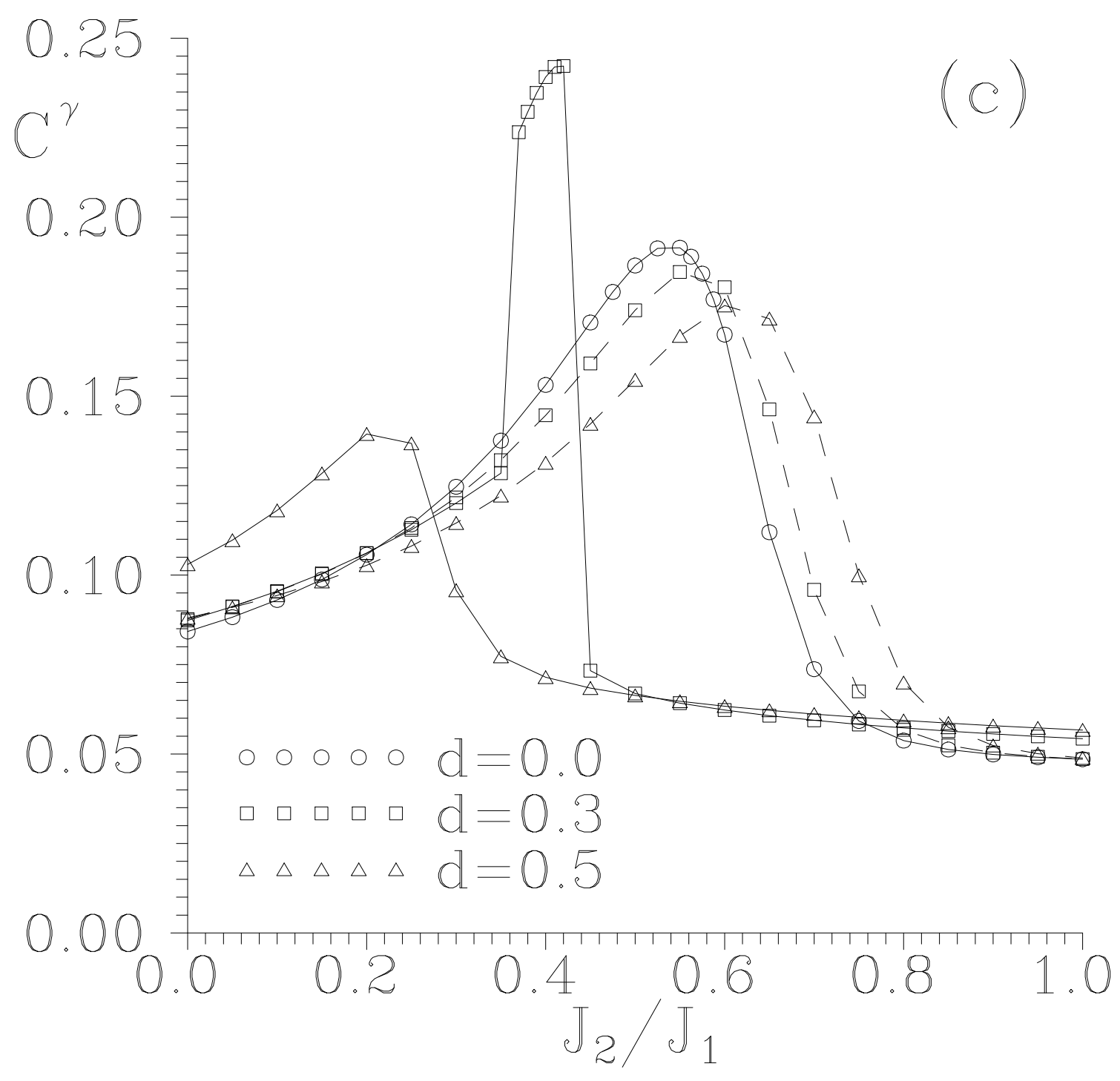

